\title{
Mapping Collagen Organization in the Human Cornea: Left and Right Eyes Are Structurally Distinct
}

\author{
Craig Boote, Sally Hayes, Mohammad Ababussin, and Keith M. Meek
}

Purpose. Aspects of the biomechanics and surface topography of fellow human corneas are known to exhibit midline symmetry, but the structural basis of these observations is poorly understood. The mechanical performance of the cornea is strongly influenced by the organization of stromal collagen fibrils. The present study was designed to examine and compare the organization of collagen fibrils in the corneal stroma of left and right eyes.

Methods. Wide-angle x-ray scattering was used to map in detail the orientation and distribution of fibrillar collagen across the cornea, limbus, and adjacent sclera of three normal human eyes, including a fellow pair, and the central 9-mm corneal region of a further four eyes.

RESULTs. Fibrillar collagen in the human cornea and limbus is arranged anisotropically, and in a highly specific manner. Left and right corneas are structurally distinct. In general, the mass distribution of preferentially aligned fibrils in the cornea appears to exhibit a degree of midline symmetry between left and right eyes.

Conclusions. Structural information, such as that presented herein, will enable a better understanding of corneal biomechanics and shape. Midline symmetry in the distribution of aligned, mechanically reinforcing collagen fibrils between left and right eyes may relate to the biomechanical and topographical enantiomorphism reported in the literature. (Invest Ophthalmol Vis Sci. 2006;47:901-908) DOI:10.1167/iovs.05-0893

$\mathrm{T}$ he cornea, together with the surrounding sclera, forms the protective outer tunic of the eye, maintaining the eyeball's shape under the action of the intraocular pressure and external physical trauma. In terrestrial vertebrates, the cornea also acts as the main refracting component, providing $\sim 70 \%$ of the total light focusing. Correct corneal curvature and transparency depend on the unique architecture of the corneal stroma, which accounts for the bulk of the tissue's thickness. In humans the stroma is composed, centrally, of $\sim 200$ stacked lamellae that lie parallel to the corneal surface. ${ }^{1}$ Each lamella contains uniformly narrow, regularly spaced collagen fibrils embedded in a hydrated matrix rich in proteoglycans, glycoproteins, other soluble proteins, inorganic salts, and keratocyte cells. Fibrils within a lamella run approximately parallel, but subtend large angles with those in adjacent lamellae. ${ }^{2}$

From the Structural Biophysics Group, School of Optometry and Vision Sciences, Cardiff University, Cardiff, Wales, United Kingdom.

Supported by Medical Research Council Grant G0001033 CCLRC Beam-time Programme Grant.

Submitted for publication July 12, 2005; revised October 6, 2005; accepted December 22, 2005.

Disclosure: C. Boote, None; S. Hayes, None; M. Abahussin, None; K.M. Meek, None

The publication costs of this article were defrayed in part by page charge payment. This article must therefore be marked "advertisement" in accordance with 18 U.S.C. $\$ 1734$ solely to indicate this fact.

Corresponding author: Keith M. Meek, Structural Biophysics Group, School of Optometry and Vision Sciences, Cardiff University, Redwood Building, King Edward VII Avenue, Cardiff CF10 3NB, UK; meekkm@cf.ac.uk.
Although corneal shape varies between individuals, it is well established that the surface topography of fellow normal human corneas exhibits a degree of nonsuperimposable midline symmetry. ${ }^{3-5}$ The structural origin of this is unknown, but it is suspected that the precise organization of stromal collagen has a role to play in determining corneal shape through a direct impact on tissue biomechanics. ${ }^{6,7}$ It has been well documented that the mechanical properties of the cornea vary across the tissue. ${ }^{8-11}$ Interlamellar cohesive strength profiles have been shown to vary anisotropically in a similar manner across fellow normal corneas, ${ }^{9}$ indicating that corresponding anisotropic reinforcing structures might exist between left and right eyes. It has been suggested ${ }^{9}$ that such an intrinsic structural feature may predispose fellow corneas to the kind of enantiomorphic ectasia reported in keratoconus. ${ }^{12}$ Moreover, certain regional differences in the cornea's mechanical properties $^{9,11}$ can be understood in terms of collagen fibril orientation models published in the literature. ${ }^{13,14}$ In general, a compelling argument may be made for the anisotropic biomechanical performance of the cornea being related to regional variations in stromal collagen organization. Computational models of corneal biomechanics are now beginning to incorporate fibril orientation data, ${ }^{15}$ with the purpose being to simulate more effectively the tissue's response to surgery. However any variational trends in collagen organization between left and right eyes, as might be expected from topographical and biomechanical considerations, remain uncharacterized, despite the potential importance of this information to our understanding of corneal mechanics and shape.

$\mathrm{X}$-ray scattering has become established as a powerful research tool for examining corneal microstructure (for a review, see Ref. 16). Scattering methods have revealed a preferential orientation of collagen fibrils in the central cornea in the superior-inferior and nasal-temporal directions. ${ }^{6,13,17}$ It has been suggested that these exist to take up the stress exerted on the cornea by the ocular motor muscles, thereby helping to preserve corneal shape. ${ }^{6,17}$ Similar methods have also determined that, toward the corneal periphery, this arrangement alters to coalesce with an annulus of fibrils that circumscribe the cornea. ${ }^{18,19}$ This annulus may help to maintain corneal curvature by mechanically reinforcing the limbus, the corneoscleral interface where the differing curvatures of the two tissues creates heightened circumferential tension. ${ }^{20}$ Recently, the technique has been used to map for the first time collagen organization across a whole cornea. ${ }^{14}$ The present study extends our knowledge by comparing the collagen organization between left and right eyes and includes structural maps of fellow corneas from a single individual.

\section{MeTHODS}

\section{Tissue Preparation}

A pair of eyeballs (donor 1) was obtained from Manchester Eye Hospital (Manchester, UK). The eyes were removed from the donor within 2 to 3 hours of death, snap frozen using liquid nitrogen and stored frozen at $-80^{\circ} \mathrm{C}$ until the time of the experiment. On removal, a scleral suture was added to mark the superior globe position. Immediately before data collection the eyes were thawed and the corneas excised, 
leaving 2 to $3 \mathrm{~mm}$ of scleral rim attached. In addition, two right eyeballs (donors 2 and 3) were provided by the Great Wall Hospital (Beijing, China). These eyes were removed (again retaining 2 to $3 \mathrm{~mm}$ of scleral rim) within 2 to 3 hours of death, sutured and stored in 10\% formalin solution until the time of data collection. (Previous x-ray experiments have revealed no significant difference between the two storage methods, in terms of the resultant wide-angle scattering data; Hayes S, personal communication, 2004.) One further right eye (donor 4) and two left eyes (donors 5 and 6) were obtained from the Bristol Eye Bank (Bristol, UK) and were harvested and prepared in an identical fashion with the eyes of donor 1 . None of the six donors had any history of ocular surgery. The tenets of the Declaration of Helsinki were adhered to throughout.

\section{Data Collection}

Wide-angle $\mathrm{x}$-ray-scattering data were collected on station 14.1 at the UK Synchrotron Radiation Source (Daresbury, UK), by using a 0.1488 -nm wavelength $\mathrm{x}$-ray beam with a square $0.2 \times 0.2 \mathrm{~mm}$ crosssection at the specimen. Specimens were wrapped in cling film and mounted inside airtight Perspex (Databank, UK) cells, to prevent tissue dehydration. X-rays were passed through the anterior face of the cornea-sclera parallel to the cornea's optical axis, and the resultant scattering data collected on a charge-coupled (CCD) x-ray detector (ADSC, Poway, CA) placed $15 \mathrm{~cm}$ behind the specimen (Fig. 1A). Scattering patterns were collected at $0.5 \times 0.5-\mathrm{mm}$ (donor 1 ) and $0.4 \times 0.4-\mathrm{mm}$ (donor 2 ) intervals across the whole tissue, with the $\mathrm{x}$-ray exposure times per data point being 45 (donor 1) and 75 (donor 2) seconds. The specimens were moved horizontally and vertically within the sample plane between exposures by means of a motorized translation stage (Newport, Newbury, UK) interfaced with the x-ray camera shutter. In addition, the central 9-mm corneal region of the four remaining eyes was examined at a resolution of 0.4 (donors 3 and 5), 0.3 (donor 4), and 0.25 (donor 6) $\mathrm{mm}$. Exposure times were 75 (donor 3), 45 (donors 4 and 5), and 30 (donor 6) seconds.

\section{Data Analysis}

Wide-angle scattering patterns from cornea and sclera arise from interference between $\mathrm{x}$-rays scattered by the regularly spaced collagen molecules making up the fibrils. ${ }^{16}$ Collagen molecules scatter x-rays in a plane normal to their long axis and, because these molecules lie nearly parallel to the fibril axis, the distribution of scattered intensity may be analyzed to yield information about the orientation of the collagen fibrils. A typical scattering pattern from the central cornea is shown in Figure 1A. It is characterized by four lobes of heightened intensity (red) arising from the predominantly orthogonal arrangement of fibrils in this part of the tissue. For each pattern the angular distribution of normalized $\mathrm{x}$-ray scatter intensity was obtained (Fig. 1B) using methods described previously. ${ }^{18}$ The scatter was divided into two components: (1) isotropic scatter from fibrils distributed equally in all directions and (2) aligned scatter from only those fibrils that are preferentially aligned (Fig. 1B). The aligned scatter was then plotted as polar vectors, introducing a $90^{\circ}$ phase shift to account for the fact that collagen molecules scatter x-rays normally to their long axis. In the resultant graph (Fig. 1C), the radial extent of the plot in any direction is proportional to the relative number of fibrils preferentially aligned in that direction. The polar plots were combined to produce a map showing the preferred orientation of fibrils over the tissue.

From the scattering patterns we were also able to compute for each sampled point in the tissue a single-intensity value giving a measure of the relative scatter from fibrillar collagen at that point. Total scatter from all fibrillar collagen was computed for each pattern by integrating the area under the angular distribution graphs (Fig. 1B). The data were then assimilated into a contour plot, as described previously, ${ }^{14}$ producing for each specimen a map showing the distribution of fibrillar collagen over the tissue. Corresponding maps were produced for only preferentially aligned fibrils by integrating the area under the aligned scatter component of the distribution graph (Fig. 1B).

\section{Results}

\section{Collagen Fibril Orientation}

By using high-intensity Synchrotron x-rays we obtained maps of collagen fibril orientation across a fellow pair of corneas (donor 1) with approximately $2 \mathrm{~mm}$ of surrounding scleral tissue. Wide-angle $x$-ray-scattering patterns were collected at

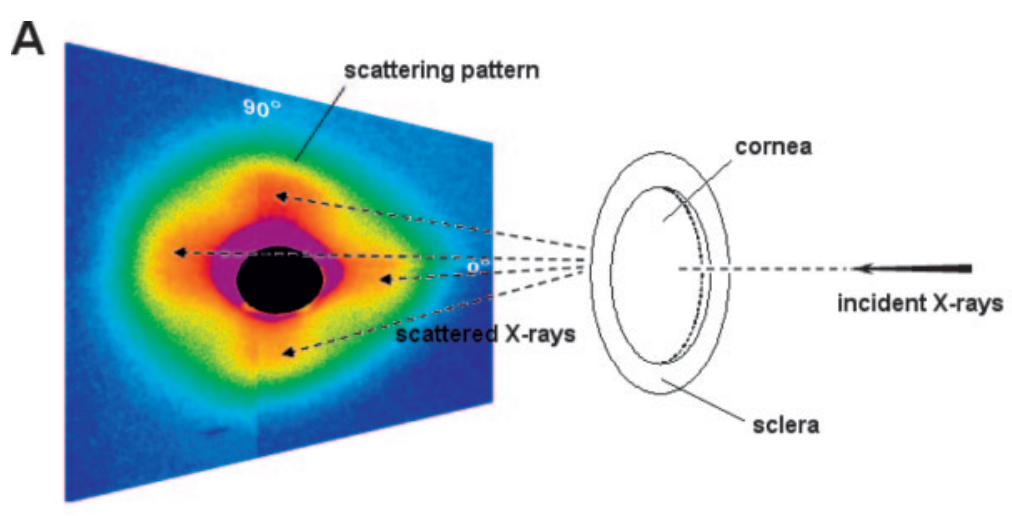

B

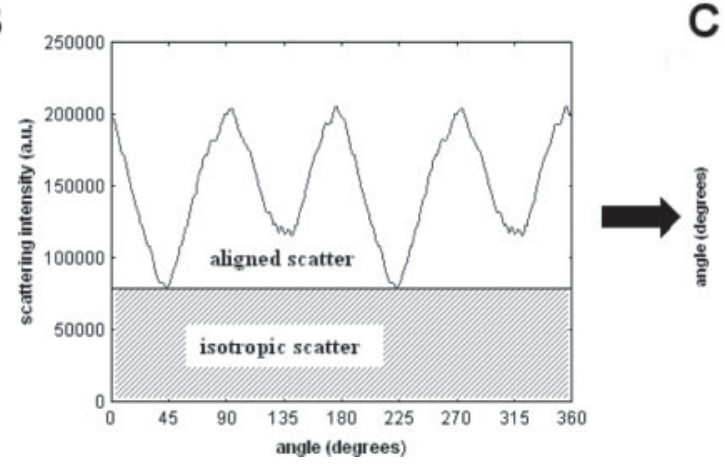

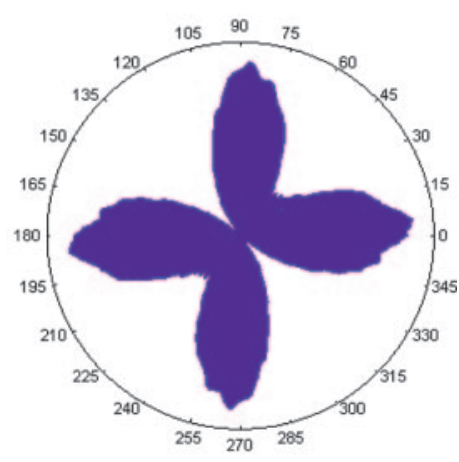

Figure 1. Wide-angle $x$-ray scattering from the cornea-sclera. (A) X-rays scattered by fibrillar collagen form a scattering pattern on a detector behind the specimen. (B) The angular distribution of scattered $x$-ray intensity is measured. (C) Scatter from preferentially aligned fibrils is converted into polar vectors. The radial extent of the resulting plot in a particular direction represents the relative number of collagen fibrils preferentially aligned in that direction. 
A

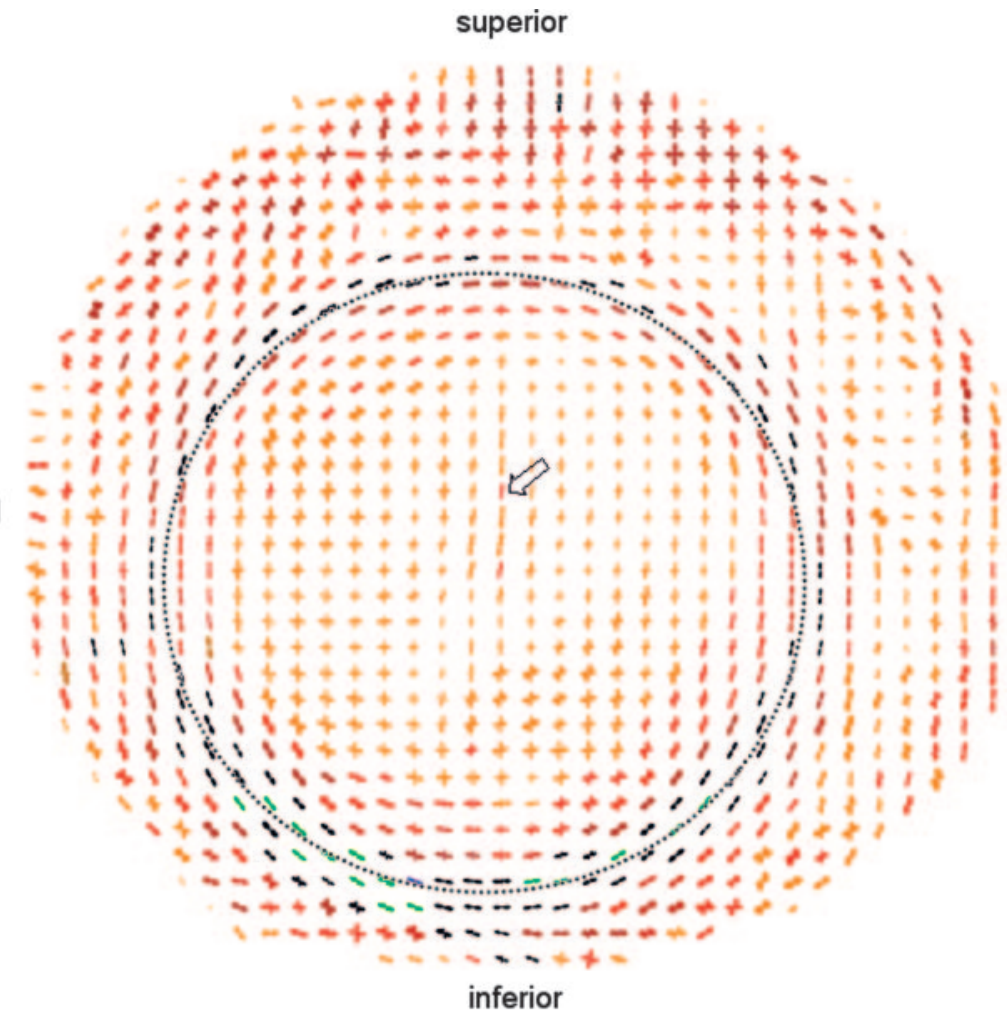

temporal

B

FIGURE 2. Preferred orientation of collagen fibrils in human cornea and sclera. (A) Left eye of donor 1, sampled at $0.5-\mathrm{mm}$ intervals. Dotted circle: the limbus. Each sampled point is represented by a polar plot, in which the radial extent of the plot in any direction is proportional to the relative number of fibrils preferentially aligned in that direction. It was necessary to scale down the larger peripheral plots. Scale factors: orange (1), red (1.5), dark red (2), black (3), green (4), blue (5). (B) Equivalent map of donor's right eye. Arrows: localized regions in both eyes where the normal orthogonal arrangement of fibrils was altered.

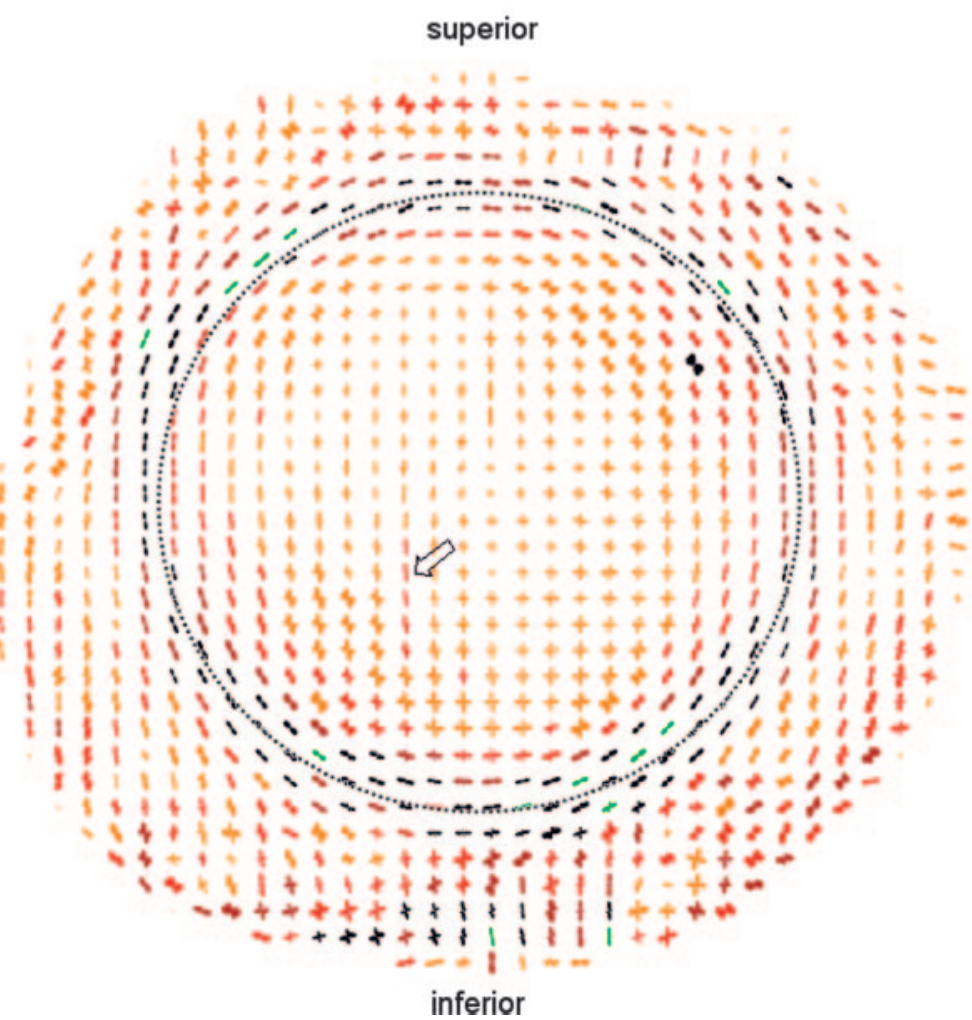

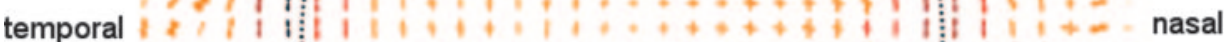

0.5-mm intervals across the whole of the cornea, limbus, and adjacent sclera. A further specimen from a right eye (donor 2) was mapped at $0.4-\mathrm{mm}$ resolution. From the scattering patterns, we were able to determine the preferred orientation of collagen fibrils, averaged through the specimen thickness, at every sampled point in the tissue. Data from donors 1 and 2 are shown in Figures 2 and 3, respectively.
Reference to the fibril orientation map for the right eye of donor 2 (Fig. 3) revealed an interesting feature of collagen organization in the sclera. The polar plots toward the periphery of the tissue clearly showed localized scleral fibrils with a preferred orientation directed radially toward the four cardinal points (designated superior, inferior, nasal, and temporal) of the globe. This feature was also noticeable to a 


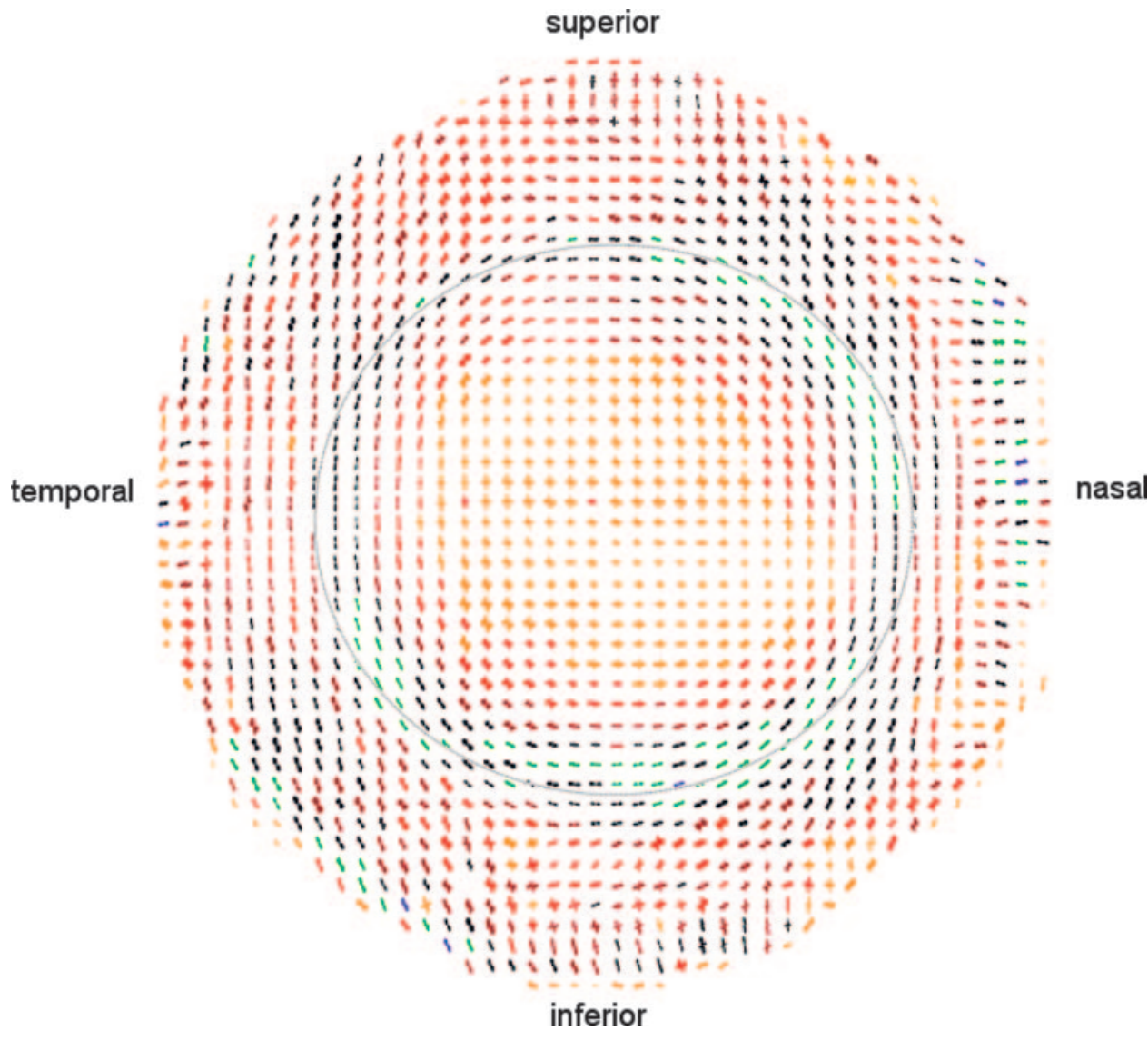

Figure 3. Map of preferred collagen fibril orientation across the right cornea-sclera of donor 2. Polar plots are shown at $0.4-\mathrm{mm}$ intervals. Scaled-down factors are as follows: orange (1), red (1.5), dark red (2), black (3), green (4), blue (5). Dotted circle: the limbus. lesser degree in the nasal and superior sclera of the left eye of donor 1 (Fig. 2A) and in the nasal and inferior sclera of the right eye (Fig. 2B). We believe it likely that these fibrils are used to anchor the sclera to the rectus muscles of the ocular motor system, which are situated at the cardinal points of the globe. $^{21}$

The polar plots in Figures 2 and 3 indicate that, in general, the central cornea of donors 1 and 2 demonstrated a preferentially orthogonal arrangement of collagen fibrils, directed along the superior-inferior and nasal-temporal meridians. This result is consistent with previous $\mathrm{x}$-ray-scattering work. ${ }^{6,13,14,17}$ There were, however, areas in both corneas of donor 1 where the orientation was locally more uniaxial (Fig. 2, arrows), being biased in favor of superior-inferior fibrils. The two observations may be reconciled if we consider that a recent study has shown that, although on average the central cornea is populated by superior-inferior and nasal-temporal fibrils in equal proportion, individual corneas may show a significant imbalance. ${ }^{6}$ A further important feature of the maps is the relative size of the polar plots in the peripheral oblique regions of the cornea. The left eye of donor 1 (Fig. 2A) clearly featured larger plots in the peripheral regions of the superior-nasal and inferior-temporal quadrants than in the two remaining quadrants. These larger plots extended 2 to $3 \mathrm{~mm}$ into the cornea relative to the center of the limbus (dotted circle) and qualitatively point to proportionally more aligned collagen in these parts of the tissue. This feature was also present in the donor's right eye (Fig. 2B) and in donor 2 (Fig. 3).

The orientation of the central corneal fibrils is clearly seen to shift toward the corneal periphery gradually (Figs. 2, 3 ), in favor of a more tangential arrangement. At the limbus (dotted circle) the plots are consistent with a circumcorneal annulus of fibrils, similar to that reported previously. ${ }^{14,18,19}$ Note also from the color of the plots that there was a much higher proportion of aligned collagen in the limbus relative to the corneal center. In agreement with previous work, ${ }^{18}$ the structure of the limbal annulus appeared asymmetricalits width and degree of fibril alignment varying with circumferential position.

\section{Collagen Distribution}

Figures $4 \mathrm{~A}, 4 \mathrm{~B}$, and $5 \mathrm{~A}$ indicate that the total scatter from fibrillar collagen remained fairly constant over the cornea (yellow contours) until approximately 1 to $1.5 \mathrm{~mm}$ from the limbus, whereupon an increase was observed (orange contours). This increase in scattering intensity was most likely due to the increased tissue thickness in this region, given that the number of stacked lamellae comprising the stroma increases more than twofold at the limbus compared with the corneal center. ${ }^{1}$ In general, scatter in the adjacent sclera demonstrated far more spatial variability than in the cornea.

Aligned collagen scatter from donor 1 is shown in Figures $4 \mathrm{C}$ and $4 \mathrm{D}$. The contours define a region of minimum scatter in the central cornea (yellow contours) which was loosely rhombic in shape, compared with the circular central region in the corresponding total scatter maps (Figs. 4A, 4B). Consistent with the collagen orientation maps (Fig. 2), the scatter was clearly elevated (orange contours) in the superior-nasal and inferior-temporal quadrants of the peripheral cornea of both eyes, causing the central rhombic region to appear skewed. This additional scatter has been proposed to arise from fibrils originating in the sclera and passing through the oblique peripheral cornea on a curved path. ${ }^{14,22}$ These so-called "anchoring" fibrils may help flatten the peripheral cornea. ${ }^{14}$ Strikingly, the resultant shape of the central aligned scatter contours in the right (Fig. 4C) 

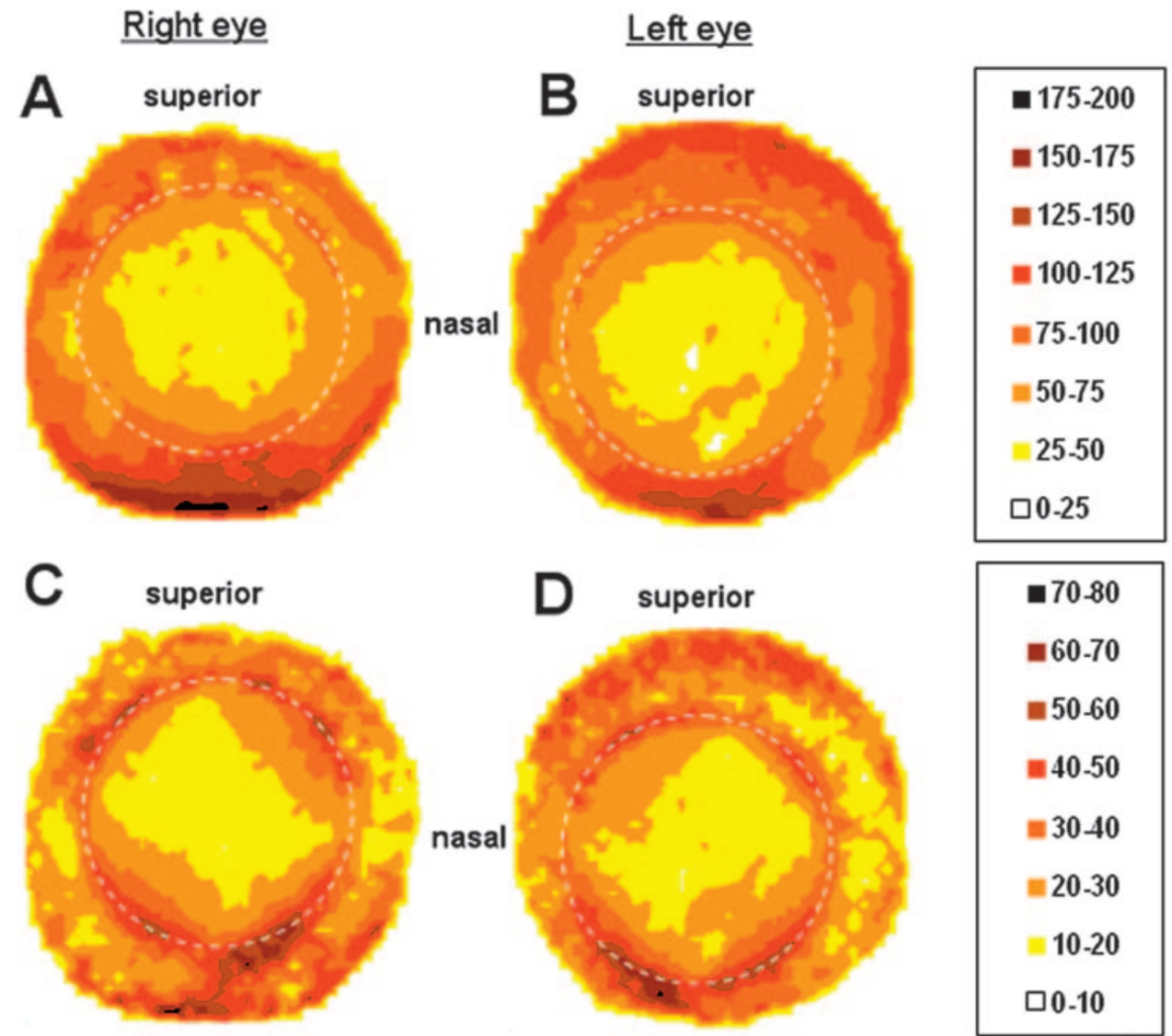

\begin{tabular}{|l|}
$\square 70-80$ \\
$\square 60-70$ \\
$\square 50-60$ \\
$=40-50$ \\
$=30-40$ \\
$=20-30$ \\
$=10-20$ \\
$\square 0-10$
\end{tabular}

FIGURE 4. Contour maps showing the distribution of collagen in human cornea and sclera. (A, B) Total fibrillar collagen $\mathrm{x}$-ray scatter from right and left eyes, respectively, of donor 1. (C, D) Scatter from only preferentially aligned collagen fibrils. (E, F) Ratio of aligned/total scatter. Scatter in arbitrary units. Dotted circle: the limbus.
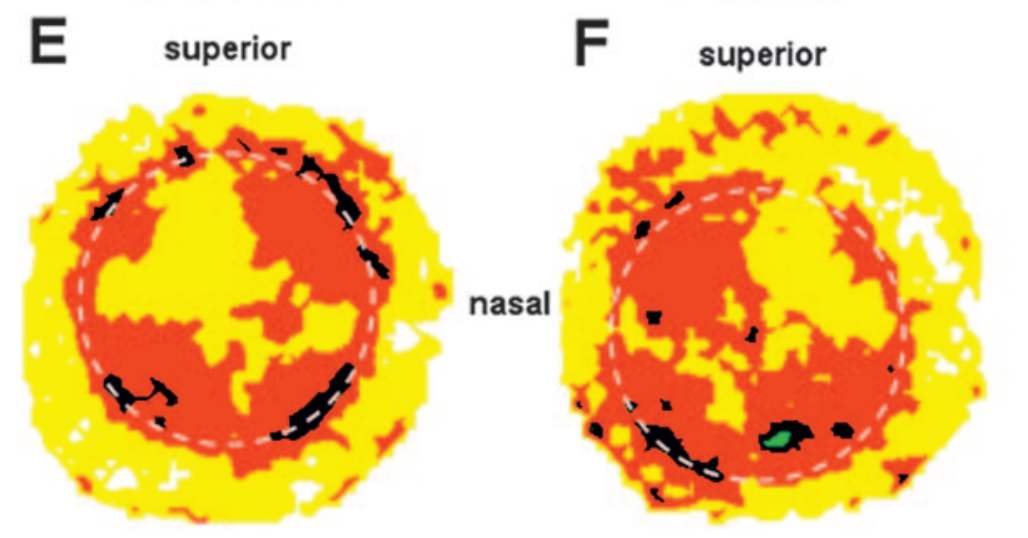

\begin{tabular}{|l|}
\hline$\square 0.8-1$ \\
$\square 0.6-0.8$ \\
$=0.4-0.6$ \\
$0.2-0.4$ \\
$\square 0-0.2$
\end{tabular}

and left (Fig. 4D) eyes exhibited a high degree of midline mirror symmetry. At the limbus (dotted line) there was a clear annulus of increased scatter intensity, which was elevated in the oblique regions compared with the cardinal points. It is further notable that maximum scatter occurred in the inferior-nasal region of the limbus in both eyes, and that overall limbal scatter is greater on the nasal side. As in the cornea, the distribution of aligned scatter around the limbus appears roughly mirrored in the fellow pair about the central body axis. Contour maps showing the ratio of aligned to total scatter are shown in Figures $4 \mathrm{E}$ and $4 \mathrm{~F}$. These were obtained by dividing the data in Figures $4 \mathrm{C}$ and $4 \mathrm{D}$, point for point, by those in Figures $4 \mathrm{~A}$ and $4 \mathrm{~B}$ respectively. The maps confirm that the apparent elevations in aligned scatter we have discussed do indeed represent genuinely higher levels of preferentially aligned collagen, as opposed to simply increases in the total amount of collagen due to, for example, variations in tissue thickness. Figures
$5 \mathrm{~B}$ and $5 \mathrm{C}$ show that all aforementioned features of the aligned collagen distribution in the right eye of donor 1 were conserved in the right eye of donor 2 . The finer sampling resolution used in the case of donor $2(0.4 \mathrm{~mm}$ instead of $0.5 \mathrm{~mm}$ ) was manifest in the improved definition of the maps in Figure 5.

To explore further the interesting observations pertaining to the distribution of aligned collagen mass, we examined the central 9-mm region of four additional corneas (two right and two left). Figure 6 shows that, although significant variation was evident between individual eyes, the characteristic distorted rhombic pattern of the aligned scatter contours was conserved. In this respect, left and right eyes may be considered structurally distinct, in that it is possible to tell a right cornea from a left by inspection of the distribution of aligned collagen alone. Notwithstanding more subtle variations between individuals, this gross structural feature appears to be an intrinsic property of the cornea. 
A

\begin{tabular}{|c|}
\hline$\square 192-216$ \\
$=168-192$ \\
$=144-168$ \\
$=120-144$ \\
$=96-120$ \\
$=72-96$ \\
$48-72$ \\
$24-48$ \\
$\square 0-24$ \\
\hline
\end{tabular}

B

$\square 91-104$
$\square 78-91$
$=65-78$
$=52-65$
$=39-52$
$=26-39$
$=13-26$
$\square 0-13$

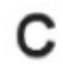

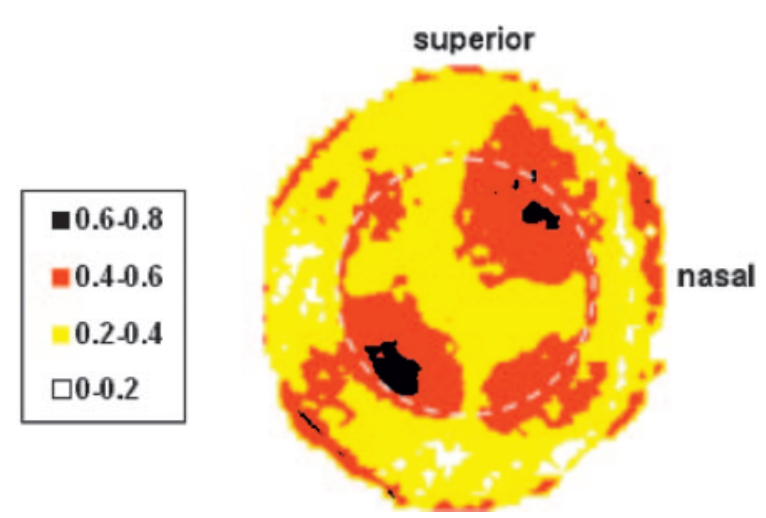
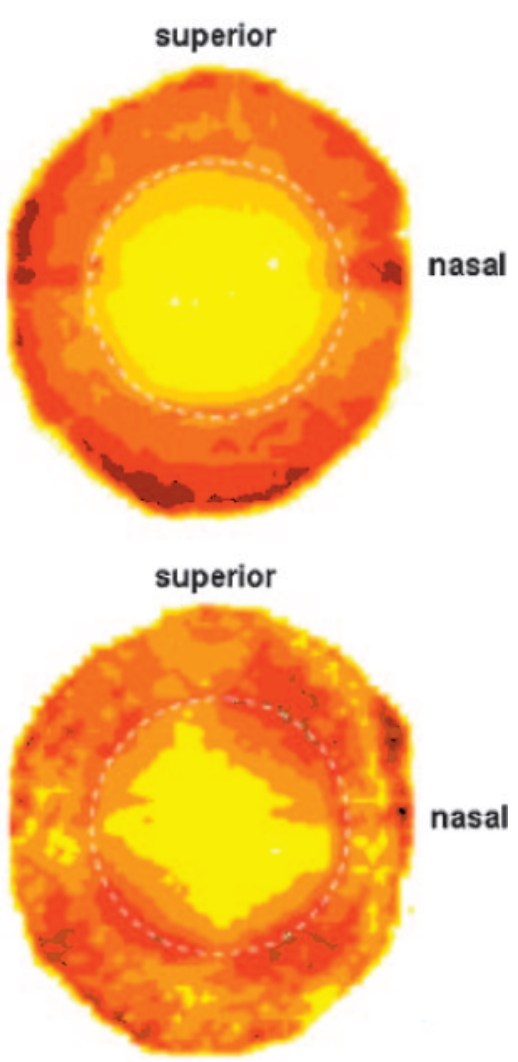

FIGURE 5. Distribution of collagen in the right cornea and sclera of donor 2. (A) Contour map of total fibrillar collagen x-ray scatter. (B) Contour map of scatter from only preferentially aligned collagen. (C) Ratio of aligned to total scatter. Scatter is expressed in arbitrary units. Dotted circle: the limbus.

\section{Discussion}

A major role of collagen fibrils in connective tissues is to provide tensile reinforcement, and knowledge of fibril orientation and distribution within a tissue is necessary for a proper understanding of the tissue's mechanical properties. $^{23,24}$ In the human eye, exactly how the arrangement of the corneal lamellae is related to mechanical performance remains to be established. In this article, we have demonstrated a population of collagen fibrils in the sclera at the four cardinal points outside the cornea that are directed toward the extraocular muscles, and it may reasonably be supposed that this collagen has a mechanical function related to eye movement. The result is consistent with the scheme originally envisioned by Kokott, ${ }^{25}$ based on lines of force measurements. Within the cornea, it is suspected that the precise organization of fibrillar collagen impacts on the tissue's shape, and thereby on the eye's refractive status. ${ }^{6,7}$ The most striking result of the present study is that the mass of preferentially aligned collagen in the corneal stroma appears to be distributed differently between left and right eyes. The characteristic rhombus-shaped contour pattern is present in every eye we have studied, and this pattern exhibited a high degree of symmetry about the midline in the fellow pair (Figs. 4C, 4D), and showed a similar leftright trend in all other specimens (Figs. 5B, 6). Of interest, variations in birefringence data across human corneas have been reported ${ }^{26}$ that demonstrate a broadly similar pattern to the observed aligned collagen distribution, although the authors were not able to comment on symmetry properties, as the specimens were not oriented. Figure 7 shows an idealized theoretical model of preferentially aligned, reinforcing fibrils in left and right human eyes based on the current data. It is possible that such an anisotropic arrangement of collagen relates to the differential flattening of the normal cornea along its various meridians. Moreover, a scheme of inherent structural symmetry such as that shown in Figure 7 may in theory help to explain the topographical enantiomorphism exhibited by fellow corneas. With this in mind, however, it is relevant to note that corneal shape is known to vary significantly from individual to individual. ${ }^{3,27}$ Further, it is unfortunate that no in vivo topographic data were available on the corneas we examined, and no direct comparison between the current data and corneal shape is therefore possible. Thus, the definitive establishment of a causal link between corneal topography and collagen organization awaits further investigation.

It is instructive to consider potential clinical implications of the current results. Intrinsic structural anisotropy, such as in the distribution of reinforcing collagen, could become a determinant of corneal shape during keratorefractive surgery. The extent of postoperative astigmatism after certain procedures depends on the size, direction, and location of the incision, ${ }^{28,29}$ and this may be related to the inhomogeneity of the stroma's fibril arrangement. ${ }^{30}$ Indeed, the outcome of computer-simulated tunnel incisions has demonstrated the benefit of using empiric data on fibril orientations to mechanically model corneal anisotropy. ${ }^{15}$ In the case of penetrating keratoplasty, the amount of surgically induced refractive error is determined by many factors, including trephination technique, ${ }^{31,32}$ suture type, ${ }^{33,34}$ and postoperative management. ${ }^{35,36}$ It is possible that collagen organization could also have a bearing on the clinical outcome, particularly given empiric evidence that graft alignment appears to affect the magnitude of astigmatism after transplant (Rapuano CJ, et al. IOVS 1995;36:ARVO Abstract 653). In addition, the results of the present study might also be relevant to LASIK surgery. Specifically, the data suggest that, during flap creation, the extent of collagen fibril disruption may depend on the direction of the microkeratome pass. However, it must be borne in mind that the current data represent an average through the full-thickness tissue. The fibril model as it stands requires extension to include structural changes with stromal depth before a conclusive argument could be made. Nevertheless, the present study may prove an integral step toward providing a fundamental structural platform on which to interpret the cornea's mechanical response to surgery. 
Right eye

A

\begin{tabular}{|c|}
\hline$=60-70$ \\
\hline$=50-60$ \\
\hline$=40-50$ \\
\hline$=30-40$ \\
\hline$=20-30$ \\
\hline$=10-20$ \\
\hline$\square 0-10$ \\
\hline
\end{tabular}

C

$\square 80-90$
$=70-80$
$=60-70$
$=50-60$
$=40-50$
$=30-40$
$=20-30$
$10-20$
$\square 0-10$

superior
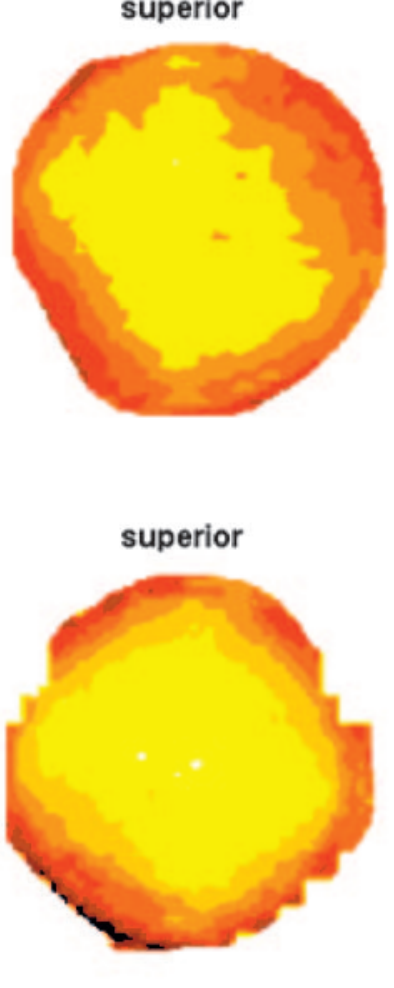

\section{Left eye}

B

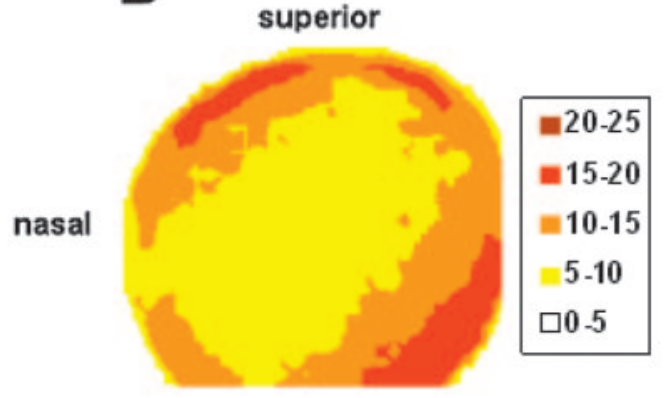

D

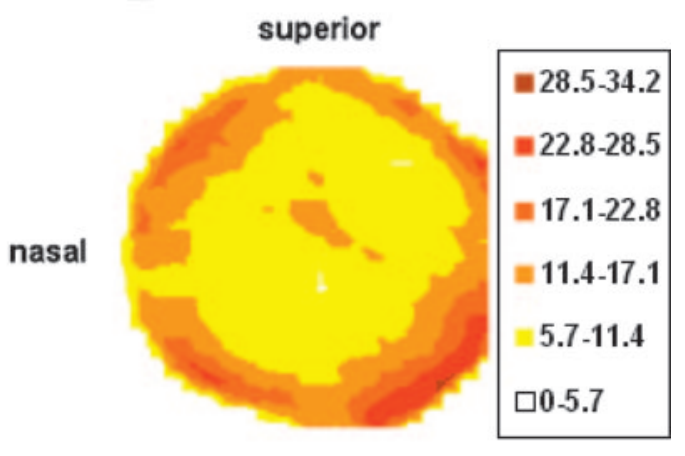

FIGURE 6. Aligned collagen scatter across the central 9-mm corneal region of (A) donor 3, right eye; (B) donor 6, left eye; (C) donor 4, right eye; (D) donor 5, left eye. Note the distorted rhombic pattern of the scatter contours, the characteristic shape of which distinguishes (A, C) right from left (B, D) corneas. Scatter is expressed in arbitrary units.

A

B

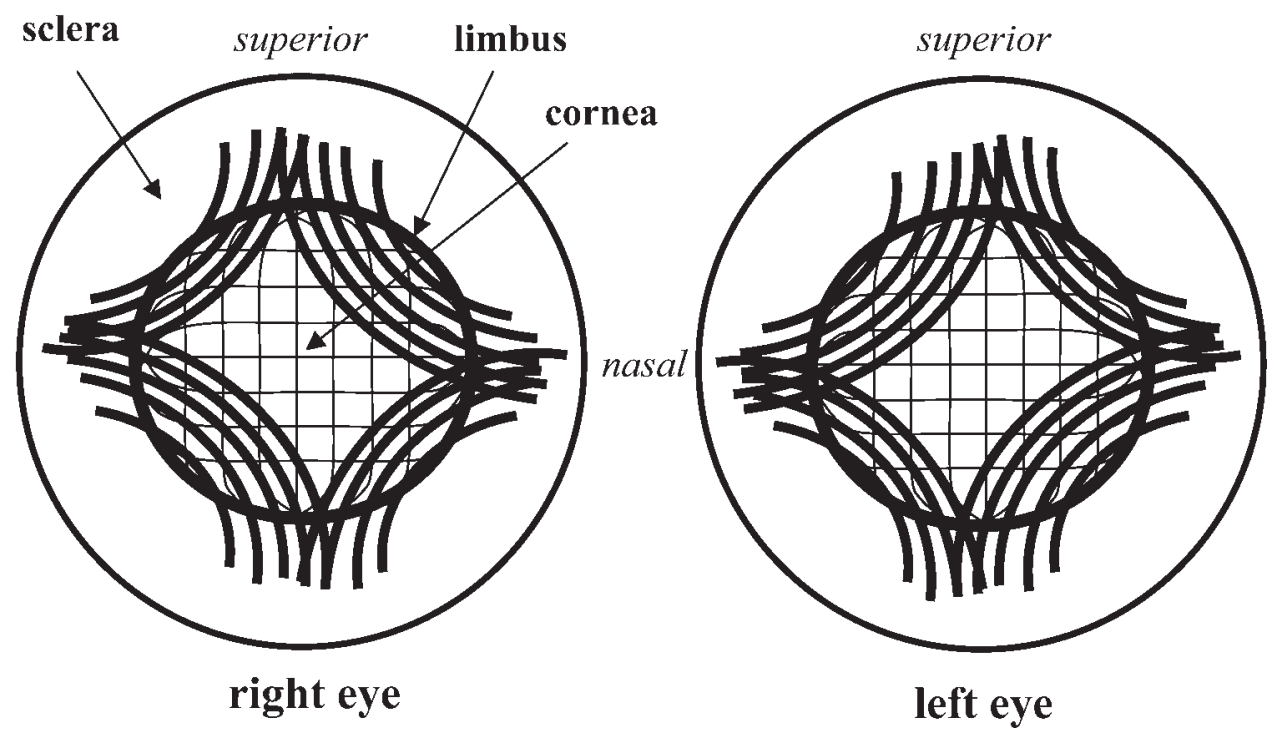

FigurE 7. Idealized theoretical model showing the directions of preferentially aligned, reinforcing collagen fibrils in the cornea, limbus, and adjacent sclera of human eyes. The fibril arrangements in (A) right and (B) left eyes are distinct, being symmetrical about the body midline. 


\section{Acknowledgments}

The authors thank Mike Macdonald and the staff of Beamline 14.1 at the UK Synchrotron X-ray Source (Daresbury, UK) for help with data collection, and Tom Kelly (Manchester Eye Hospital, UK), Yifei Huang (Great Wall Hospital, Beijing, China), and Val Smith (Bristol Eye Bank, UK) for provision of specimens.

\section{References}

1. Maurice DM. The structure and transparency of the corneal stroma. J Physiol. 1957;136:263-286.

2. Komai Y, Ushiki T. The three-dimensional organization of collagen fibrils in the human cornea and sclera. Invest Ophthalmol Vis Sci. 1999;32:2244-2258.

3. Dingeldein SA, Klyce SD. The topography of normal corneas. Arch Ophthalmol. 1989;107:512-518.

4. Smolek MK, Klyce SD, Sarver EJ. Inattention to nonsuperimposable midline symmetry causes wavefront analysis error. Arch Ophthalmol. 2002;120:439- 447 .

5. Serrao S, Lombardo G, Lombardo M. Differences in nasal and temporal responses of the cornea after photorefractive keratectomy. J Cataract Refract Surg. 2005;31:30-38.

6. Boote C, Dennis S, Meek KM. Lamellar orientation in human cornea in relation to mechanical properties. J Struct Biol. 2005; 149:1-6.

7. Meek KM, Tuft SJ, Huang Y, et al. Changes in collagen orientation and distribution in keratoconus corneas. Invest Ophthalmol Vis Sci. 2005;46:1948-1956.

8. Smolek MK. Interlamellar adhesive strength in human eyebank corneas. Invest Ophthalmol Vis Sci. 1990;31:1087-1095.

9. Smolek MK. Interlamellar cohesive strength in the vertical meridian of human eye bank corneas. Invest Ophthalmol Vis Sci. 1993; 34:2962-2969.

10. Hjordtal JO. Regional elastic performance of the human cornea. J Biomechanics. 1996;29:931-942.

11. Shin TJ, Vito RP, Johnson LW, McCarey BE. The distribution of strain in the human cornea. J Biomechanics. 1997;30:497-503.

12. Wilson SE, Lin DTC, Klyce SD. Corneal topography of keratoconus. Cornea. 1991;10:2-8.

13. Meek KM, Blamires T, Elliott GF, Gyi TJ, Nave C. The organisation of collagen fibrils in the human corneal stroma: a synchrotron X-ray diffraction study. Curr Eye Res. 1987;6:841-846.

14. Aghamohammadzadeh H, Meek KM. X-ray scattering used to map the preferred collagen orientation in the human cornea and limbus. Structure. 2004;12:249-256.

15. Pinsky PM, van der Heide D, Chernyak D. Computational modeling of mechanical anisotropy in the cornea and sclera. J Cataract Refract Surg. 2005;31:136-145.

16. Meek KM, Quantock AJ. The use of X-ray scattering techniques to determine corneal ultrastructure. Prog Retin Eye Res. 2001;20:95137.
17. Daxer A, Fratzl P. Collagen fibril orientation in the human corneal stroma and its implications in keratoconus. Invest Ophthalmol Vis Sci. 1997;38:121-129.

18. Newton RH, Meek KM. Circum-corneal annulus of collagen fibrils in the human limbus. Invest Ophthalmol Vis Sci. 1998;39:11251134 .

19. Newton RH, Meek KM. The integration of the corneal and limbal fibrils in the human eye. Biophys J. 1998;75:2508-2512.

20. Maurice DM. The cornea and sclera. In: Davson $\mathrm{H}$, ed. The Eye. Orlando, FL: Academic Press; 1969:489-600.

21. Wolff E. Anatomy of the Eye and Orbit. 9th ed. London: Arnold; 1997.

22. Meek KM, Boote C. The organization of collagen in the corneal stroma. Exp Eye Res. 2003;78:503-512.

23. Hukins DWL. Collagen orientation. In Hukins DWL, ed. Connective Tissue Matrix. London: Macmillan; 1984:210-240.

24. Hukins DWL, Aspden RM. Composition and properties of connective tissues. Trends Biochem Sci. 1985;10:260-264.

25. Kokott W. Über mechanisch-funkionelle Strukturen des Auges. Albrecht von Graefes Arch Ophthalmol. 1938;138:424-485.

26. Jaronski JW, Kasprzak HT. Linear birefringence measurements of the in vitro human cornea. Ophthalmic Physiol. 2003;23:361369.

27. Bogan SJ, Waring GO III, Ibrahim O, Drews C, Curtis L. Classification of normal corneal topography based on computer-assisted videokeratography. Arch Ophthalmol. 1990;108:945-949.

28. Kohnen T. Corneal shape changes and astigmatic aspects of scleral and corneal tunnel incisions. J Cataract Refract Surg. 1987;23: 301-302.

29. Wirbelauer C, Anders N, Pham DT, Wollensak J. Effect of incision location on preoperative oblique astigmatism after scleral tunnel incision. J Cataract Refract Surg. 1997;23:365-371.

30. Meek KM, Newton RH. Organization of collagen fibrils in the corneal stroma in relation to mechanical properties and surgical practice. J Refract Surg. 1999;15:695-699.

31. Girard LJ, Eguez I, Esnaola N, Barnett L, Maghraby A. Effect of penetrating keratoplasty using grafts of various sizes on keratoconic myopia and astigmatism. J Cataract Refract Surg. 1988;14: 541-547.

32. Mahjoub SB, Au YK. Astigmatism and tissue shape disparity in penetrating keratoplasty. Ophthalmic Surg. 1990;21:187-190.

33. Musch DC, Meyer RF, Sugar A, Soong HK. Corneal astigmatism after penetrating keratoplasty: the role of suture technique. $O p h$ thalmology. 1989;96:698-703.

34. Assil KK, Zarnegar SR, Schanzlin DJ. Visual outcome after penetrating keratoplasty with double continuous or combined interrupted and continuous suture wound closure. Am J Ophthalmol. 1992;114:63-71.

35. Binder PS. Selective suture removal can reduce post keratoplasty astigmatism. Ophthalmology. 1985;92:1412-1416.

36. Van Meter WS, Gussler JR, Soloman KD, Wood TO. Postkeratoplasty astigmatism control: single continuous suture adjustment versus selective interrupted suture removal. Ophthalmology. 1991;98:177-183. 